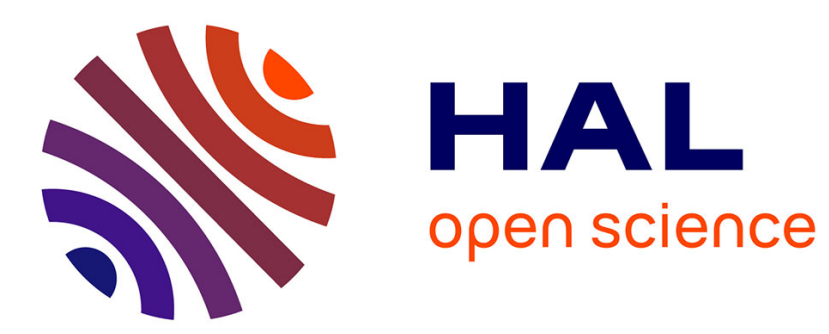

\title{
Estimation of angular difference between tomographic projections taken at unknown directions in $3 \mathrm{D}$
}

Minh Son Phan, Etienne Baudrier, Loïc Mazo, Mohamed Tajine

\section{To cite this version:}

Minh Son Phan, Etienne Baudrier, Loïc Mazo, Mohamed Tajine. Estimation of angular difference between tomographic projections taken at unknown directions in 3D. 2015 IEEE International Conference on Image Processing (ICIP), Sep 2015, Quebec City, France. 10.1109/ICIP.2015.7350762 . hal-01910167

\section{HAL Id: hal-01910167 \\ https://hal.science/hal-01910167}

Submitted on 31 Oct 2018

HAL is a multi-disciplinary open access archive for the deposit and dissemination of scientific research documents, whether they are published or not. The documents may come from teaching and research institutions in France or abroad, or from public or private research centers.
L'archive ouverte pluridisciplinaire HAL, est destinée au dépôt et à la diffusion de documents scientifiques de niveau recherche, publiés ou non, émanant des établissements d'enseignement et de recherche français ou étrangers, des laboratoires publics ou privés. 


\title{
ESTIMATION OF ANGULAR DIFFERENCE BETWEEN TOMOGRAPHIC PROJECTIONS TAKEN AT UNKNOWN DIRECTIONS IN 3D
}

\author{
Minh Son Phan, Étienne Baudrier, Lö̈c Mazo, Mohamed Tajine \\ ICube, University of Strasbourg, CNRS \\ 300 Bd Sébastien Brant - CS 10413 - 67412 ILLKIRCH, FRANCE
}

\begin{abstract}
This paper deals with the estimation of angular difference between two tomographic projections belonging to a set of projections taken at unknown directions. The proposed method extends our former work from 2D to 3D. The method is potential for many applications such as projection refinement or projection classification, which are important in the process of tomographic reconstruction. Unlike to common line based angular estimation, the proposed method does not need reference projections. Our method relies on the selection of projection neighbors with local adaptive thresholds, the calculus of the angular difference for neighboring projections by using properties of moments. The accuracy and the robustness of our method are shown on a test database including 50 3D gray-level images at different resolutions and with different levels of noise.
\end{abstract}

Index Terms - tomography, unknown direction, angular difference, Euclidean distance, invariant moments

\section{INTRODUCTION}

Tomographic reconstruction for unknown direction projections is a well-known problem in various domains such as cryo electron microscopy or medical imaging. During the process of reconstruction, the distance between two projection vectors is measured and the Euclidean distance is often used. For example, in $[1,2]$, the authors compute the Euclidean distances between the neighboring projections in order to classify the projections and estimate their directions. In [3], after generating an initial reconstruction model, Van Heel computes the Euclidean distance between the projections of the reconstruction model and those acquired from data for the purpose of refining the reconstruction model. The Euclidean distance is also used in [4] for simultaneously estimating the projection directions and reconstructing the object.

However, the calculus of the Euclidean distance between two projections has a wide variation even if the two projections are close. This may lead to errors when classifying or refining projections. On the other hand, our previous work in 2D presented a method that can estimate the angular difference between two projections by using the properties of mo- ments [5]. The variation of the angular difference estimated from this method is very smaller than the Euclidean distance. This enables us to extend our work to 3D for estimating the angular difference between the neighboring projections. The organisation of the paper is as follows. We first provide some background notions in Sec. 2. Then, the estimation problem of the angular difference is presented in Sec. 3. The performance of our method is carried out in Sec. 4. Finally, we conclude in Sec. 5.

\section{BACKGROUND NOTIONS}

\subsection{Rotation in 3D}

We first present the rotation convention used in this paper. The rotation of a vector in $3 \mathrm{D}$ is defined as the rotation of this vector around the three axes $x, y$ and $z$ by the three angles $\varphi, \theta$ and $\psi$. It can be represented as the multiplication of the three rotation matrices $\mathrm{R}_{\varphi}^{x}, \mathrm{R}_{\theta}^{y}$ and $\mathrm{R}_{\psi}^{z}$. For example, the product

$$
\mathrm{R}_{\varphi, \theta, \psi}=\mathrm{R}_{\varphi}^{x} \mathrm{R}_{\theta}^{y} \mathrm{R}_{\psi}^{z}
$$

represents a rotation of a vector around the $z, y$ and $x$ axis by the angles $\psi, \theta$ and $\varphi$, respectively. We also note that

$$
\mathrm{R}_{\varphi, \theta, \psi}^{-1}=\mathrm{R}_{-\psi}^{z} \mathrm{R}_{-\theta}^{y} \mathrm{R}_{-\varphi}^{x}=\mathrm{R}_{-(\psi, \theta, \varphi)} .
$$

\subsection{Projection}

The projection of an object can be obtained by first rotating the coordinates system using the rotation matrix $\mathrm{R}_{\varphi, \theta, \psi}$, then project the object onto the new $(x O y)$ plane. This procedure is similar to the rotation of the object using the rotation matrix $\mathrm{R}_{-(\psi, \theta, \varphi)}$ and project the object onto the $(x O y)$ plane. We define the projection as follows

Definition 1 (Projection). Let $f: \mathbb{R}^{3} \rightarrow[0,1]$ be a measurable function with compact support and let $\varphi, \theta, \psi \in \mathbb{R}$. The projection $\mathcal{P}_{f}(\varphi, \theta, \psi)$ of $f$ in the direction $(\varphi, \theta, \psi)$ is defined by

$$
\mathcal{P}_{f}(\varphi, \theta, \psi)(x, y)=\int_{\mathbb{R}} f\left(\rho_{-(\psi, \theta, \varphi)}(x, y, z)\right) \mathrm{d} z,
$$


where

$$
\rho_{\psi, \theta, \varphi}(x, y, z)=\left(\operatorname{R}_{\psi, \theta, \varphi}\left(\begin{array}{l}
x \\
y \\
z
\end{array}\right)\right)^{T} .
$$

Note that $\mathcal{P}_{f}(\varphi, \theta, \psi)=\mathcal{P}_{f}(\varphi+\pi, \theta+\pi, \psi+\pi)$. Thus we can assume that $\varphi, \theta, \psi \in[-\pi / 2, \pi / 2]$.

We also present the direction vector of the projection, that is the unit vector in the new $z$ axis direction as follows

$$
\begin{aligned}
v_{\mathcal{P}}(\varphi, \theta) & =\mathrm{R}_{\varphi, \theta, \psi}(0,0,1)^{T} \\
& =(\sin \theta,-\sin \varphi \cos \theta, \cos \varphi \cos \theta)^{T} .
\end{aligned}
$$

Note that the direction vector $v_{\mathcal{P}}(\varphi, \theta)$ does not depend on the angle $\psi$.

\subsection{Moment of projection}

We recall that for a function $g: \mathbb{R}^{2} \rightarrow \mathbb{R}$, the moment of $g$ with order $(c, d)$, where $c, d \in \mathbb{N}$ is given by

$$
\mu_{c, d}(g)=\int_{\mathbb{R}} \int_{\mathbb{R}} x^{c} y^{d} g(x, y) \mathrm{d} x \mathrm{~d} y .
$$

For a function $f: \mathbb{R}^{3} \rightarrow \mathbb{R}$ and $\varphi, \theta, \psi \in \mathbb{R}$, we note $\mu_{f, c, d}(\varphi, \theta, \psi)=\mu_{c, d}\left(\mathcal{P}_{f}(\varphi, \theta, \psi)\right)$ a moment with order $(c, d)$ of the projection $\mathcal{P}_{f}(\varphi, \theta, \psi)$.

The moments of projection in $2 \mathrm{D}$ are trigonometric polynomials as a function of projection direction as shown in [6]. In $3 \mathrm{D}$, we obtain the following result

Proposition 1 (Trigonometric polynomial of moment). Let $f: \mathbb{R}^{3} \rightarrow[0,1]$ be a measurable function with compact support, let $\varphi, \theta, \psi \in \mathbb{R}$ and $c, d \in \mathbb{N}$. The moment of the projection $\mathcal{P}_{f}(\varphi, \theta, \psi)$ of the function $f$, with the order $(c, d)$, in the direction $(\varphi, \theta, \psi)$ is a trigonometric polynomial as a function of projection direction.

In the following, for simplicity, we write $\mathcal{P}$ and $\mu_{c, d}$ instead of $\mathcal{P}_{f}$ and $\mu_{f, c, d}$.

\section{ESTIMATION OF THE ANGULAR DIFFERENCE}

\subsection{Problem of estimating the angular difference}

We consider a function $f$ whose centroid is at $(0,0,0)$, a set of unknown directions $\Theta=\left\{\left(\varphi_{i}, \theta_{i}, \psi_{i}\right) \mid 1 \leq i \leq n\right\} \subseteq$ $\left[-\pi / 2, \pi / 2\left[{ }^{3}, n \in \mathbb{N}\right.\right.$. We set $\Pi=\left\{\mathcal{P}\left(\varphi_{i}, \theta_{i}, \psi_{i}\right) \mid 1 \leq\right.$ $i \leq n\}$ a set of projections associated to $\Theta$ and $\mathrm{V}=$ $\left\{v_{\mathcal{P}}\left(\varphi_{i}, \theta_{i}\right) \mid 1 \leq i \leq n\right\}$ a set of direction vectors of $\Pi$. Our purpose is to estimate the angular difference between two neighboring projections from the set of projections $\Pi$. The following formula calculates the angular difference between two projections $\mathcal{P}$ and $\mathcal{P}^{\prime}$, that is the inner product between two direction vectors $v_{\mathcal{P}}$ and $v_{\mathcal{P}^{\prime}}$

$$
\mathrm{d}_{\text {ang }}\left(\mathcal{P}, \mathcal{P}^{\prime}\right)=\arccos \left(v_{\mathcal{P}} \cdot v_{\mathcal{P}^{\prime}}\right)
$$

In order to calculate the angular difference from the equation (4), the direction vector must be calculated. There are two well-known approaches to the direction estimation problem. The first approach is based on the common line technique as shown in [7]. The main idea is that two projections share one line in common which is perpendicular to the direction vectors of the two projections. Thus, using the two common lines between three projections enables us to find the direction vectors of these projections. However, the common line technique is time consuming and may lead to accumulative errors when detecting the false common lines. We therefore employ the second approach that estimates the direction vector of projection by analysing the relationship between the moments of projections and the moments of the 3D function $[8,9]$. The method of moments is fast and does not lead to the accumulation of errors as in the first approach. We have developed from [8] the formula of direction vector as follows

$$
v_{\mathcal{P}}(\varphi, \theta)=\left(v_{\mathcal{P}}^{1}, v_{\mathcal{P}}^{2}, v_{\mathcal{P}}^{3}\right)^{T}
$$

where :

$$
\begin{aligned}
& v_{\mathcal{P}}^{1}= \pm\left(\frac{1}{a}\left(\mu_{\text {max }}-\mu_{2,0}(\varphi, \theta, \psi)\right)\left(\mu_{\text {max }}-\mu_{0,2}(\varphi, \theta, \psi)\right)\right)^{1 / 2}, \\
& \text { with } a=\left(\mu_{\text {max }}-\mu_{\text {med }}\right)\left(\mu_{\text {max }}-\mu_{\text {min }}\right), \\
& v_{\mathcal{P}}^{2}= \pm\left(\frac{1}{b}\left(\mu_{\text {med }}-\mu_{2,0}(\varphi, \theta, \psi)\right)\left(\mu_{\text {med }}-\mu_{0,2}(\varphi, \theta, \psi)\right)\right)^{1 / 2}, \\
& \text { with } b=-\left(\mu_{\text {max }}-\mu_{\text {med }}\right)\left(\mu_{\text {med }}-\mu_{\text {min }}\right) \\
& v_{\mathcal{P}}^{3}=\left(\frac{1}{c}\left(\mu_{\text {min }}-\mu_{2,0}(\varphi, \theta, \psi)\right)\left(\mu_{\text {min }}-\mu_{0,2}(\varphi, \theta, \psi)\right)\right)^{1 / 2}, \\
& \text { with } c=\left(\mu_{\text {max }}-\mu_{\text {min }}\right)\left(\mu_{\text {med }}-\mu_{\text {min }}\right) \text { and } \\
& \mu_{\text {max }}=\max _{(\varphi, \theta, \psi) \in \mathbb{R}} \max \left(\mu_{2,0}(\varphi, \theta, \psi), \mu_{0,2}(\psi, \theta, \varphi)\right), \\
& \mu_{\text {med }}=\min _{(\varphi, \theta, \psi) \in \mathbb{R}} \max \left(\mu_{2,0}(\varphi, \theta, \psi), \mu_{0,2}(\psi, \theta, \varphi)\right), \\
& \mu_{\text {min }}=\min _{(\varphi, \theta, \psi) \in \mathbb{R}} \min \left(\mu_{2,0}(\varphi, \theta, \psi), \mu_{0,2}(\psi, \theta, \varphi)\right) .
\end{aligned}
$$

There are four possible direction vectors in (5) due to the unknown signs. These four direction vectors are on four different octants of the coordinates system. However, we can obtain a unique solution in (5) if we make the assumption that both of the projections are in the same octant. This is the case for most neighboring projection pairs if there is a large number of projections and if the distribution of projections is uniform. Thus, we can forget the problem of signs in (5) by simply setting $v_{\mathcal{P}}=\left(\left|v_{\mathcal{P}}^{1}\right|,\left|v_{\mathcal{P}}^{2}\right|, v_{\mathcal{P}}^{3}\right)^{T}$. Then the angular difference formula between two neighboring projections is modified as follows

$$
\mathrm{d}_{\text {ang }}\left(\mathcal{P}, \mathcal{P}^{\prime}\right)=\arccos \left(\sum_{i=1}^{3}\left|v_{\mathcal{P}}^{i} v_{\mathcal{P}^{\prime}}^{i}\right|\right)
$$

The problem of searching the neighbors of each projection is presented in the following section. 


\subsection{Searching for the neighbors of projection}

In [5], we have developed a method for finding the neighbors of each projection in $2 \mathrm{D}$. The principle of this method is that, for each moment of a given projection, with a given order, we compute a threshold that allows us to find at least one moment corresponding to a neighbor of the projection. The method can also be applied in 3D. However, the moments are not used directly since the projection can be rotated around its direction vector by an arbitrary angle. This changes the value of moments and leads to errors for neighboring searching. Instead, we use $\mathrm{Hu}$ moments [10], for their invariance to the rotation of projection. These invariant moments can be calculated easily from of the (geometric) moments. Note that the (geometric) moments are trigonometric polynomials (Proposition 1) and it is known that the set of trigonometric polynomials is a ring. Thus, these invariant moments are also trigonometric polynomials.

$\mathrm{Hu}$ moments are now applied for selecting the neighbors of a given projection. Since, these moments are nonmonotonic, close moments are not synonymous with close direction vectors of projections. Thus, for each $\mathrm{Hu}$ moment $\mathcal{M}(\varphi, \theta)$ of a given projection, we find an interval $\mathcal{M}(\varphi, \theta) \pm \varepsilon^{p}(\varphi, \theta)$ in which can be found, with some chosen probability $p \in[0,1]$, at least one moment corresponding to a neighbor of the projection. Assuming that the number of projections is sufficient large and uniformly distributed on demi-sphere. We first find for each angle $\lambda \in\{\varphi, \theta\}$, an interval $\lambda \pm \delta_{\lambda}$ in which there is at least one angle $\lambda^{\prime}$. Since the distribution of projections is uniform, we can obtain the formula of $\delta_{\lambda}$ as follows

$$
\delta_{\lambda}=\left\{\begin{array}{ll}
\frac{\pi}{2}\left(1-(1-p)^{\frac{1}{\sqrt{n}-1}}\right), & \text { if } \lambda=\varphi \\
\frac{1}{\sqrt{\left(v_{\mathcal{P}}^{2}\right)^{2}+\left(v_{\mathcal{P}}^{3}\right)^{2}}}\left(1-(1-p)^{\frac{1}{\sqrt{n}-1}}\right), & \text { if } \lambda=\theta
\end{array} .\right.
$$

We also note that $\mathrm{Hu}$ moments $\mathcal{M}(\varphi, \theta)$ are trigonometric polynomials. Then, by using Bernstein's inequality [11] for trigonometric polynomials, we have

$$
\varepsilon^{p}(\varphi, \theta) \approx k\left(\delta_{\varphi}+\delta_{\theta}\right) \max _{(\varphi, \theta) \in \mathbb{R}}|\mathcal{M}(\varphi, \theta)|,
$$

where $k$ is the order of $\mathcal{M}(\varphi, \theta)$.

In particular, when $\mathcal{M}=\mu_{2,0}+\mu_{0,2}$, a better value of $\varepsilon^{p}$ can be obtained as follows

$$
\varepsilon^{p}(\varphi, \theta) \approx 2\left(\xi_{\varphi} \delta_{\varphi}+\xi_{\theta} \delta_{\theta}\right)
$$

where

$$
\begin{aligned}
& \xi_{\varphi}=\left(\left(\mathcal{M}(\varphi, \theta)-\mu^{\text {med }}\right)\left(\mu^{\text {max }}+\mu^{\text {med }}-\mathcal{M}(\varphi, \theta)\right)\right)^{1 / 2}, \\
& \xi_{\theta}=\left(\left(\mathcal{M}(\varphi, \theta)-\mu^{\text {max }}\right)\left(\mu^{\text {max }}+\mu^{\text {med }}+\mu^{\text {min }}-\mathcal{M}(\varphi, \theta)\right)\right)^{1 / 2} .
\end{aligned}
$$

In conclusion, (7) and (8) allow us to find sets of projection neighbors for distinct moment orders. Then, the final result is obtained by intersecting these set of projection neighbors.
Remark 1. In Section 3, the exact values of $\mu^{\max }, \mu^{\text {med }}$ and $\mu^{\text {min }}$ used in (5), (7) and (8) are not known. We can only estimate $\mu^{\max }, \mu^{\text {med }}$ and $\mu^{\text {min }}$ from the finite set of projections $\Pi$. However, if the angular difference $d_{\text {ang }}$ between two neighboring projections is small in probability, then we can assume that the errors on $\mu^{\text {max }}, \mu^{\text {med }}$ and $\mu^{\text {min }}$ less than $\left(\mathrm{d}_{\mathrm{ang}}\right)^{2}$ and do not modify the formulae obtained above.

\section{RESULTS}

We apply our method on a set of 50 phantom images of different resolutions $\left(32^{3}, 64^{3}, 128^{3}\right.$ and $256^{3}$ voxels $)$ as shown in Fig. 1. We first generate randomly a set of 400 triplets $(\varphi, \theta, \psi)$, where $\varphi, \theta, \psi \in[-\pi / 2, \pi / 2[$. Then, a set of projections $\mathcal{P}(\varphi, \theta, \psi)$ is computed for each image. We next find the neighbors of each projection $\mathcal{P}(\varphi, \theta, \psi)$ by using the Eq. (7) and (8). Finally, the angular differences $d_{\text {ang }}$ between the neighboring projections are estimated by the Eq. (6). The Euclidean distance between these neighboring projections are also computed. Our objective is to measure the dispersion of our estimated angular difference $\widehat{\mathrm{d}}_{\text {ang }}$ (resp. the Euclidean distance) with respect to the exact angular difference $d_{a n g}$. We use the variance-to-mean ratio (VMR) presented in [5] for measuring the dispersion. The VMR should be lower than 0.5 for a small dispersion. As seen in Table. 1, the VMR of our estimated angular difference $\widehat{\mathrm{d}}_{\text {ang }}$ is very smaller than 0.5 for all image resolutions, whereas VMR of the Euclidean distance is higher than 1. An example for the dispersion of the Euclidean distance and the $\widehat{\mathrm{d}}_{\text {ang }}$ is illustrated in Fig. 2. We see that the dispersion of the Euclidean distance ( $x$ axis) with respect to $\mathrm{d}_{\text {ang }}$ ( $y$ axis) is greater than the $\widehat{\mathrm{d}}_{\text {ang }}$.

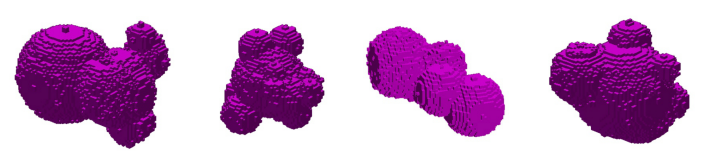

Fig. 1: Examples from the 3D database

Table 1: Mean and standard deviation of the VMR for the Euclidean distance and the $\widehat{\mathrm{d}}_{\mathrm{ang}}$, according to the image resolutions.

\begin{tabular}{|c|c|c|}
\hline Resolution & Euclidean distance & $\widehat{\mathbf{d}}_{\text {ang }}$ \\
\hline $32^{3}$ voxels & $1.03 \pm 0.40$ & $0.06 \pm 0.03$ \\
\hline $64^{3}$ voxels & $0.98 \pm 0.32$ & $0.05 \pm 0.01$ \\
\hline $128^{3}$ voxels & $1.09 \pm 0.34$ & $0.05 \pm 0.02$ \\
\hline $256^{3}$ voxels & $0.83 \pm 0.37$ & $0.05 \pm 0.03$ \\
\hline
\end{tabular}

The robustness of our method against noise is also evaluated. A white noise with a given SNR is added to the projections as seen in Fig. 3. The results of estimating $d_{\text {ang }}$ are 


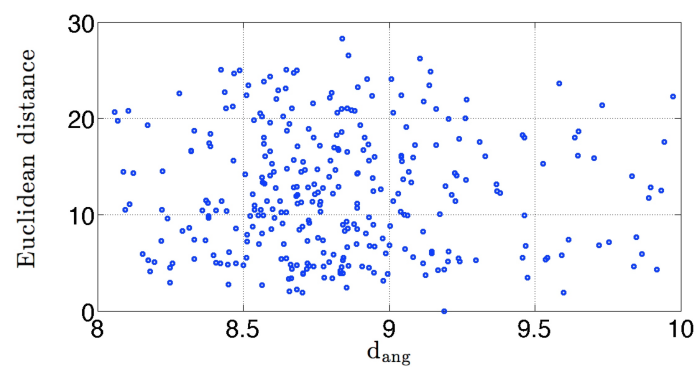

(a)

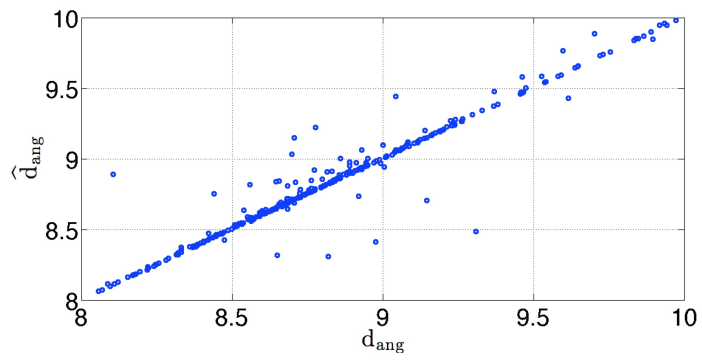

(b)

Fig. 2: Dispersion of the Euclidean distance (a) and our estimated angular difference (b), from an image of size $64^{3}$ voxels.

shown in Fig. 4 for the images of $256^{3}$ voxels. The mean of VMR is still lower than 0.5 at the SNR $>10 \mathrm{~dB}$.

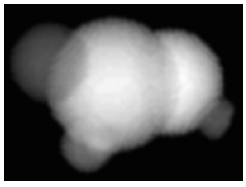

(a) Without noise

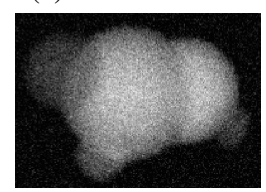

(c) $10 \mathrm{~dB}$

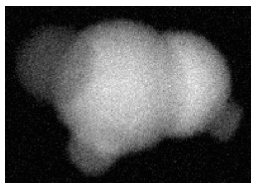

(b) $15 \mathrm{~dB}$

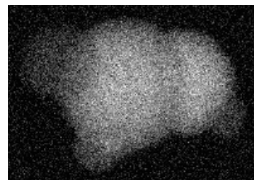

(d) $5 \mathrm{~dB}$
Fig. 3: Examples of noisy projections.

We further applied our method to a real image TAF7 of $160^{3}$ voxels as shown in Fig.5. 400 noisy projections are generated from the TAF7 and the Wiener filter is used to denoise the projections. The positive results are obtained at the $\mathrm{SNR} \geq 10 \mathrm{~dB}$ with the VMR $\leq 0.5$.

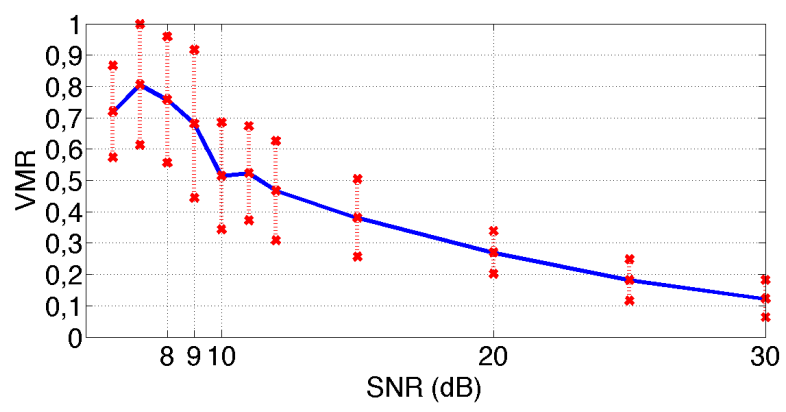

Fig. 4: Mean and standard deviation of the VMR for $\widehat{\mathrm{d}}_{\text {ang }}$ against white noise, from the images of $256^{3}$ voxels.

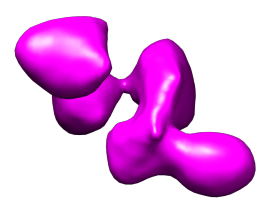

Fig. 5: Real 3D image TAF7.

\section{CONCLUSION}

This paper extends our new method to the 3D case. We deal with the problem of estimating the angular difference between two neighboring projections taken at unknown directions. We first find the neighbors of each projection based on local adaptive thresholds for the moments of projections. Then formula of estimating the angular difference between two neighboring projections is given. Our method has been evaluated on a set of 3D images at different resolutions and with different levels of noise. The results demonstrated that our estimated angular difference performs better than the Euclidean distance and is robust at moderate levels of noise. Our method can be applied for many applications such as projection refinement, projection classification, etc.

Finding the neighboring projections and estimating the angular differences between them are important for the following step of our work. This allows us to calculate the angular difference between any two projections by constructing a neighborhood graph whose edges connect the neighboring projections, weighted by the estimated angular differences and then using the shortest path algorithm on the graph. This work will be completed in the future.

\section{REFERENCES}

[1] R. R. Coifman, Y. Shkolnisky, F. J. Sigworth, and A. Singer, "Graph laplacian tomography from unknown random projections," IEEE Trans Image Process, vol. 17, no. 10, pp. 1891-1899, Oct. 2008. 
[2] Y. Fang, M. Sun, S. Vishwanathan, and K. Ramani, "sLLE: Spherical locally linear embedding with applications to tomography," 2011 IEEE Conference on Computer Vision and Pattern Recognition (CVPR), pp. 1129-1136, Jun. 2011.

[3] M. Van Heel, "Multivariate statistical classification of noisy images (randomly oriented biological macromolecules)," Ultramicroscopy, vol. 13, no. 1-2, pp. 165$183,1984$.

[4] B. Ben Cheikh, É. Baudrier, and G. Frey, "A tomographical reconstruction method from unknown direction projections for 2D gray-level images," in 2014 IEEE 11th Int Symp on Biomed Imaging (ISBI), Apr. 2014, pp. 209-212.

[5] M.-S. Phan, É. Baudrier, L. Mazo, and M. Tajine, "Angular difference measure between tomographic projections taken at unknown directions in 2d," in 2014 IEEE International Conference on Image Processing (ICIP), Oct. 2014, pp. 1738-1742.

[6] F. Natterer, The Mathematics of Computerized Tomography. SIAM, Jun. 2001.

[7] M. Van Heel, "Angular reconstitution: a posteriori assignment of projection directions for 3D reconstruction," Ultramicroscopy, vol. 21, no. 2, pp. 111-123, 1987.

[8] D. B. Salzman, "A method of general moments for orienting 2D projections of unknown 3D objects," Comput Vision Graph, vol. 50, no. 2, pp. 129-156, May 1990.

[9] A. B. Goncharov and M. S. Gelfand, "Determination of mutual orientation of identical particles from their projections by the moments method," Ultramicroscopy, vol. 25, no. 4, pp. 317-327, 1988.

[10] M.-K. Hu, "Visual pattern recognition by moment invariants," IRE Transactions on Information Theory, vol. 8, no. 2, pp. 179-187, Feb. 1962.

[11] S. Bernstein, "Sur l'ordre de la meilleure approximation des fonctions continues par des polynômes de degré donné," Mémoires de l'Académie Royale de Belgique, vol. 2, no. 4, pp. 1-103, 1912. 